Revue d'histoire de l'Amérique française

REVUE D.HISTOIRE DE L'AMÉRIQUE FRANÇAISE

\title{
Le nationalisme canadien-français et les classes sociales
}

\section{Gérald Fortin}

Volume 22, numéro 4, mars 1969

URI : https://id.erudit.org/iderudit/302824ar

DOI : https://doi.org/10.7202/302824ar

Aller au sommaire du numéro

Éditeur(s)

Institut d'histoire de l'Amérique française

ISSN

0035-2357 (imprimé)

1492-1383 (numérique)

Découvrir la revue

Citer cet article

Fortin, G. (1969). Le nationalisme canadien-français et les classes sociales. Revue d'histoire de l'Amérique française, 22(4), 523-535.

https://doi.org/10.7202/302824ar d'utilisation que vous pouvez consulter en ligne.

https://apropos.erudit.org/fr/usagers/politique-dutilisation/ 
Gérald Fortin

\section{LE NATIONALISME CANADIEN-FRANÇAIS}

ET

LES CLASSES SOCIALES

Pierre Dandurand

Commentaire

NDLR - Nous reproduisons le texte intégral des communications sur "le nationalisme au Canada français aux XIXe et XXe siècles" présentées lors du Colloque des étudiants en histoire de l'Université Laval, les 31 janvier et 1er février derniers, sauf celle du professeur Maurice Séguin dont on pourra se faire une idée par son étude publiée au Boréal Express sous le titre: L'idée d'indépendance au Québec: genèse et historique. Ces communications sont suivies d'un résumé substantiel des commentaires. 


\section{Gérald Fortin}

Maître en Sociologie de Laval, docteur en Sociologie de Cornell University en 1956.

Professeur titulaire au Département de sociologie de l'Université Laval.

Principales publications :

An Analysis of the ideology of a French Canadian Nationalist Magazine 1917-1954, thèse de doctorat présentée à Cornell University.

"Changements sociaux et transformations idéologiques: deux exemples", dans Recherches sociographiques, LV (mai-août 1963).

"Le Québec, une société à la recherche d'elle-même", dans Recherches sociographiques, no 1 (1967).

\section{Pierre Dandurand}

Licencié en Philosophie et maître en Sociologie de l'Université de Montréal. Docteur de l'Université de Paris.

Professeur au Département de Sociologie de l'Université de Montréal.

\section{Auteur de :}

Analyse de lidéologie nationaliste fondée sur LE DEVOIR. Thèse présentée en vue de l'obtention de la maîtrise en Sociologie. 


\section{LE NATIONALISME CANADIEN-FRANÇAIS ET LES CLASSES SOCIALES}

Tenter de rapprocher les concepts de nationalisme et de classe sociale semble à première vue une entreprise relativement futile. En effet, le nationalisme, pour autant qu'il tente de mobiliser toutes les énergies d'un groupe ethnique ou d'une société en vue de sa libéralisation ou de son autodétermination, doit nécessairement minimiser les différences qui existent à l'intérieur de la nation. Le nationalisme tend ainsi à nier ou à diminuer les différences de classe dans la société pour mettre l'accent sur la similitude de tous les membres de la nation, sur la grande famille ou communauté qu'elle constitue. Les idéologies nationalistes sont ordinairement très peu explicites sur les phénomènes de classe sociale. En fait, ces idéologies traitent des classes sociales uniquement lorsque, pour une raison ou pour une autre, certaines classes se détachent du courant nationaliste. Ainsi, j'ai pu montrer dans ma thèse de doctorat que l'action nationale se préoccupait de la classe ouvrière seulement lorsque les syndicats se détachaient de l'idéologie nationaliste traditionnelle et attaquaient les patrons canadiensfrançais. Le corporatisme qui permettait d'unir dans une même organisation ouvriers et patrons est, comme on le sait, la formule idéale souhaitée par le nationalisme canadien-français traditionnel au moment de son expression la plus articulée, c'est-à-dire dans les années 1930-1940.

Négation de la lutte de classe et de l'importance de la division de la société en classes, le nationalisme ne peut toutefois réussir à faire disparaître ce phénomène. Si le nationalisme s'inscrit dans une société concrète, il a à prendre position par rapport à certains sous-groupes de cette société, il a surtout à présenter un projet de société qui suppose une certaine division 
du pouvoir et, pour autant, une certaine relation entre les sousgroupes. Ce projet de société contient donc toujours au moins implicitement une définition des classes et des relations qui doivent exister entre ces classes dans la nation à réaliser.

De plus dans une société concrète, le nationalisme est proposé et soutenu par certains groupes plus que par d'autres. La visée nationaliste des différentes classes devient donc un phénomène social important susceptible de nous éclairer de façon supplémentaire sur le genre de société désirée par le projet nationaliste. En effet, de même que pour le nationalisme les classes sociales et leur dynamisme sont un moyen pour atteindre l'objectif désiré, de même pour les classes sociales le nationalisme peut être un moyen plus ou moins efficace pour atteindre l'objectif de classe. Dans sa démarche pour garder le pouvoir ou pour s'en emparer, une classe sociale cherche à convaincre les autres classes de la légitimité de ses prétentions au pouvoir. Le nationalisme, quand il présente un projet de société et une certaine organisation des classes, peut devenir une source de légitimité très importante, du fait même qu'il minimise le problème du pouvoir interne et obscurcit les rapports de classe. Le pouvoir accordé à une certaine classe est ainsi justifié par le bien de l'ensemble de la société, par les liens inaltérables de l'ethnie ou de l'histoire. Plus le projet nationaliste légitimera le pouvoir d'une classe, plus cette classe aura tendance à promouvoir le nationalisme et à s'en faire le défenseur. Inversement moins une classe trouvera de légitimation de son pouvoir dans le nationalisme, moins elle y adhérera; dans certains cas elle pourrait même être fortement opposée au nationalisme. Alors que le nationalisme tend à nier la division en classes, les idéologies de classes, elles, tendent à nier le nationalisme et à chercher l'union mondiale des individus appartenant à une même classe. Il s'agit donc théoriquement de deux principes opposés de lecture de la société et d'action politique. Cependant ces deux principes deviennent l'un pour l'autre des moyens utiles et peuvent ainsi se réconcilier au niveau de la praxis d'une société concrète. En particulier les classes qui veulent conserver le pouvoir ou qui sont tout près de s'en emparer pourront avoir 
tendance à être nationalistes afin de justifier de façon globale leur revendication. Ces classes chercheront alors à insérer dans le projet nationaliste leur propre vision de la société et leur propre projet de société.

Ainsi l'analyse du projet ou des projets nationalistes canadiens-français et des groupes qui ont été les animateurs principaux de ce nationalisme peut-elle devenir un élément important dans la compréhension de la structure sociale de notre société et de l'évolution de cette structure.

Une première forme de nationalisme va apparaître au Canada dans la première moitié du XIXe siècle qui va atteindre son point maximum avec la Confédération et réapparaître à la fin du XIXe siècle avec Henri Bourassa. Il ne s'agit pas à proprement parler d'un nationalisme canadien-français, mais plutôt d'un nationalisme canadien axé surtout sur l'émancipation de la métropole. La colonie, le Canada, veut aboutir à l'autodétermination, à la parité avec la mère patrie.

Cependant la composante nationaliste de ce mouvement paraît moins importante que sa composante de libéralisme politique qui cherche en même temps que la libération de la métropole l'établissement du gouvernement responsable et de la démocratie politique. Sans doute les deux aspects sont-ils très fortement liés, car accéder à la responsabilité gouvernementale, c'est se libérer de l'emprise des gouverneurs et de leur conseil qui n'ont de compte à rendre qu'à la métropole. Cependant il me semble que la dimension du libéralisme politique est la plus importante du moins dans les débats et les écrits. Je dois avouer néanmoins que, n'étant pas un spécialiste du XIXe siècle, il est possible que cette affirmation soit davantage une impression personnelle qu'un fait véritablement établi. Je dois avouer de plus mon ignorance relative quant au projet de société véhiculé par les libéraux nationalistes du XIXe siècle. Pour autant toutefois que ce projet semble s'opposer à l'aristocratie et à la bourgeoisie commerçante d'Angleterre, l'accession au gouvernement responsable me semble être l'équivalent canadien de la Révolution française, c'est-à-dire l'accession au pouvoir de la 
bourgeoisie industrielle et commerçante. Qui sont en effet les supporteurs principaux de ce projet sinon la bourgeoisie intellectuelle canadienne-française et la bourgeoisie commerciale canadienne-anglaise ? Du côté canadien-français, tous ceux-là des professions libérales qui ont de la difficulté à s'intégrer dans la société de langue française aussi bien que dans la société de langue anglaise; du côté canadien-anglais, sans doute quelques professionnels aussi, mais un groupe important de commerçants et de capitalistes naissants.

Grâce aux changements constitutionnels, la bourgeoisie canadienne-anglaise paraît avoir réussi à asseoir son pouvoir de façon satisfaisante, puisque, après ces réformes, son nationalisme diminue considérablement s'il ne disparaît pas complètement. Au plus voit-on apparaître, par la suite et jusqu'aujourd'hui, une forme mitigée de nationalisme qui s'oppose non plus à l'Angleterre mais aux États-Unis. Il s'agit d'ailleurs alors strictement d'un nationalisme culturel dont les points forts tendent à coïncider avec certaines commissions royales d'enquête. Du côté du Canada anglais, une fois que le nationalisme a rempli son rôle de légitimation, les phénomènes de classes prennent le dessus. Les capitalistes aussi bien que les ouvriers s'uniront avec leurs collègues des Etats-Unis dans leur lutte pour le pouvoir.

Il n'en va pas de même du côté canadien-français. À peine le nationalisme canadien a-t-il porté fruit que naît le nationalisme canadien-français. $\bar{A}$ la fin du XIXe siècle va se cristalliser ce qu'il est maintenant convenu d'appeler le nationalisme canadienfrançais traditionnel. Établissant le pouvoir du capitalisme canadien, le gouvernement responsable apporte relativement peu à la bourgeoisie canadienne-française qui est surtout une bourgeoisie professionnelle.

Cette élite libérale avait pu se lier de façon temporaire avec la bourgeoisie commerçante anglaise afin de conquérir le gouvernement responsable. Les succès mêmes de cette alliance dans l'établissement du capitalisme canadien enlevaient toutefois à l'élite libérale canadienne-française ses chances de domination 
dans l'ensemble de la société canadienne. Auxiliaires utiles pendant la lutte, les professionnels canadiens-français redevenaient des parasites inutiles après la victoire.

Les romans du XIXe siècle insistent presque tous sur cette situation d'aliénation du jeune professionnel canadien-français, en particulier du jeune avocat. Ces mêmes romans nous indiquent aussi la solution entrevue par cette élite à la recherche du pouvoir. Il s'agit de s'éloigner des Anglais et d'aller fonder, dans du territoire neuf, une nouvelle société symbolisée par une nouvelle paroisse. Les professionnels rencontraient ainsi la vieille utopie ruraliste du clergé sous le Régime français. Cette utopie d'ailleurs avait continué à exister chez le clergé après la Conquête, d'autant plus que les besoins de la nouvelle métropole exigeaient que le Canada devienne une colonie agricole au lieu d'être une colonie basée sur le commerce de la fourrure. L'alliance qui semble s'être réalisée à la fin du XIXe siècle et au début du $\mathrm{XXe}$ siècle entre les professionnels et le clergé est peut-être moins basée sur le pouvoir effectif du clergé que sur la recherche du pouvoir par les professionnels. Moins qu'un pouvoir le clergé possédait un projet de société apte à donner aux professionnels la place qu'il croyait leur revenir. Il faudrait peut-être se poser de nouveau la question: qui du clergé ou des professionnels a le plus utilisé l'autre groupe?

Porte-étendard d'un projet de société, Monseigneur Paquet et ses collègues ont peut-être été davantage les dindons de la farce que les véritables moteurs du nationalisme traditionnel.

Quel est donc ce projet de société que le nationalisme traditionnel a imposé comme image du Canada français jusqu'en 1950 ? Celle d'une société française et catholique pouvant s'autodéterminer parallèlement à la société canadienne-anglaise urbaine, industrielle et bourgeoise. Cette société devait donc être rurale et agricole. Elle devait non seulement condamner mais ignorer la ville, l'industrie, le commerçant, l'industriel, l'ouvrier. Son seul point d'appui solide était la classe agricole. Ces cultivateurs qui devaient former la majorité de la population devaient être égaux entre eux. De même que la société devait être auto- 
suffisante, de même chaque famille devait être capable de pourvoir à ses besoins. Les possibilités de production devaient donc être également réparties entre toutes les familles et seul le hasard pouvait permettre à certains individus de surpasser les autres. Ce hasard toutefois ne conférait aucun statut particulier à ceux qui avaient le mieux réussi, la richesse étant un attribut de la société qu'on voulait éviter. Par ailleurs ce peuple égal devait être gouverné par une élite éclairée. Sans doute le clergé faisait-il partie de cette élite éclairée, mais le concept d'élite était élargi à l'ensemble des professions libérales et des gens instruits. Le bon Pasteur était couvert aussi bien de la soutane que de la toge.

C'est cette possibilité pour les clercs en général de s'emparer complètement du pouvoir qui rendait le projet de société si intéressant pour la bourgeoisie professionnelle canadiennefrançaise. Aussi ne faut-il pas s'étonner de voir que les principaux collaborateurs de l'Action française et de l'Action nationale sont des journalistes, des professeurs d'université, des prêtres et des professionnels dans la pratique privée (la plupart d'ailleurs résident à Montréal et n'ont d'autres contacts avec le milieu rural que la poésie ou les souvenirs d'enfance).

Parallèlement à ce nationalisme rural proposé par l'élite professionnelle, a toujours existé durant cette période un nationalisme économique. Le projet est ici radicalement opposé à celui du nationalisme majoritaire. Il s'agit pour les Canadiens français de s'emparer de la petite et de la moyenne industrie, de s'installer dans les villes et de concurrencer à leur niveau les capitalistes canadiens-anglais ou étrangers. Ce nationalisme économique qui prend naissance à la fin du XIXe siècle est surtout fort durant les années ' 20 et va complètement disparaître durant les années '30 et '40. Il est proposé par des économistes et des hommes d'affaires. Nationalisme de la petite bourgeoisie capitaliste canadienne-française qui cherche à augmenter son pouvoir menacé par le capitaliste canadien-anglais, cette idéologie, bien qu'elle se soit parfois alliée avec le nationalisme traditionnel, est toujours demeurée minoritaire et sans grand 
résultat. Faible chandelle vascillante, elle est à l'image de notre bourgeoisie capitaliste.

Le nationalisme traditionnel a permis au Québec le règne des avocats et des professions libérales. Il l'a permis d'autant plus facilement que ceux qui détenaient le pouvoir ont réussi à répandre l'idée que le pouvoir appartenait réellement au clergé.

Assurant le pouvoir des professions libérales, le nationalisme traditionnel voulait convertir à l'agriculture une société qui n'avait jamais été réellement agricole, et qui de jour en jour le devenait de moins en moins. L'écart entre le projet et la réalité devait finir par faire éclater le projet lui-même. C'est la classe ouvrière que le nationalisme traditionnel négligeait complètement qui devait porter le coup de grâce aux projets ruralistes. Guidée par des intellectuels devenus ouvriéristes après un passage aux sciences sociales ou dans l'action catholique spécialisée, la C.T.C.C. cessait d'être une union de boutique contrôlée par l'idéologie nationale pour devenir un syndicat agressif n'hésitant pas à s'allier à l'occasion avec les syndicats internationaux. En 1953, André Laurendeau jette le cri d'alarme dans l'Action nationale: les ouvriers ne sont plus nationalistes, la majorité de la population ne croit plus au projet qu'on lui propose; il faut redéfinir le projet. Le cri d'alarme vient cependant trop tard: le vieux nationalisme ne peut plus se redéfinir, le règne des avocats, même s'il se poursuit jusqu'en 1960, est déjà fini.

Le nationalisme canadien-français n'était pas mort pour autant, et il devait réapparaître avec une vigueur nouvelle dans les années 1960. Le néo-nationalisme est cependant loin d'être unitaire. En analysant ses différentes formes, on saisit davantage les forces qui sont en présence dans notre société contemporaine et on peut peut-être prévoir quelle sera notre société de demain.

Un premier fait à noter, c'est que, dans toutes ses versions différentes, le néo-nationalisme contient un projet général commun. Le Québec sera une société urbaine et industrielle, si possible la plus urbaine et la plus industrielle des sociétés nord- 
américaines. Elle aura de plus résolu les problèmes majeurs de la société nord-américaine anglo-saxonne et aliénée. Ce qui rend difficile l'analyse du néo-nationalisme contemporain, c'est que le projet va rarement plus loin pour indiquer comment cette nouvelle société va se réaliser et s'organiser concrètement. En particulier, il est très rare que le projet spécifie la structure de pouvoir qui organisera cette société urbaine et industrielle. Pour découvrir le contenu concret du projet ou des projets, il nous faut donc recourir surtout à l'examen des groupes qui proposent ce néo-nationalisme.

Il est à noter que ni les cultivateurs ni les ouvriers ne sont des éléments importants dans cette renaissance du nationalisme canadien-français. Tout au plus peut-on percevoir que la C.S.N. essaie d'utiliser son caractère québecois aux fins de recrutement ou dans les conflits intersyndicaux. Cette tactique d'ailleurs ne réussit pas toujours très bien. Sans doute cultivateurs et ouvriers sont-ils trop loin d'une prise effective du pouvoir pour chercher à mobiliser le reste de la population avec des arguments de type nationaliste.

Le groupe le plus important à proposer le nouveau nationalisme est sans doute celui des techniciens, ingénieurs, économistes, sociologues, etc., qui constituent la nouvelle technocratie gouvernementale et privée. Il s'agit de la nouvelle élite produite par notre société depuis 20 ans et qui s'oppose à l'élite libérale traditionnelle. Cette élite nouvelle prend conscience de sa capacité à contrôler le contexte urbain et industriel, mais doit entrer en concurrence avec une technocratie anglo-saxonne déjà établie depuis plusieurs années. Un État séparé lui apparaît comme un moyen indispensable pour s'assurer les postes-clés qu'elle recherche. En donnant à l'État le rôle principal dans le développement de la société, les technocrates veulent à la fois assurer leur propre pouvoir et se donner un instrument qui leur permette de réaliser la société de bien-être qu'ils désirent instaurer. Cette société par ailleurs sera relativement égalitaire: biens et services seront redistribués à la masse afin de lui permettre d'atteindre un niveau de vie et même un mode de vie relativement humain et 
valable. Le pouvoir cependant appartiendra aux technocrates seuls capables d'interpréter les besoins de la masse et seuls capables de mettre en branle les moyens pour satisfaire ces besoins.

Un deuxième groupe important de néo-nationalistes est constitué par la petite bourgeoisie économique et une partie de l'élite libérale détrônée. Ce nationalisme est souvent dit de droite et veut réaliser le nationalisme économique des années 1920. Pour ce groupe, comme pour le premier, l'État a un rôle primordial à jouer dans la construction de la société québecoise. Devant l'impossibilité de créer un capitalisme canadien-français capable de concurrencer le capitalisme américain, ce groupe propose que l'État devienne le capitalisme principal. Ce capitalisme d'État permettrait l'établissement d'une structure industrielle assez forte pour que les capitaux privés canadiens-français puissent consolider la moyenne et la petite industrie. L'État ne serait plus ainsi au service de la masse mais au service de la classe bourgeoise.

Un troisième groupe semble composé d'intellectuels et d'étudiants venant surtout de la philosophie et des lettres. Ce groupe lui-même se divise en deux. On pourrait qualifier un premier sous-groupe de nationalistes linguistiques. Le projet ne semble pas dépasser la sauvegarde de la langue par tous les moyens possibles. Ces nationalistes sont ordinairement prêts à s'allier avec n'importe quel autre groupe à condition que ces groupes reconnaissent la primauté absolue de la langue.

Le deuxième sous-groupe est peut-être celui qui a le projet le plus défini et a alimenté pendant quelques années la revue Parti-Pris. Ce groupe veut par le nationalisme donner le pouvoir aux ouvriers, alliant ainsi marxisme et nationalisme. Contrairement cependant au véritable marxiste pour qui le nationalisme se situe seulement au niveau des moyens, le nationalisme apparaît ici comme une fin et est placé sur un pied d'égalité avec le socialisme. Le jugement est difficile à porter sur ce mariage non-orthodoxe. Tout au plus peut-on constater que ce "nationalo- 
socialisme" a eu très peu de succès auprès des ouvriers euxmêmes.

Un dernier groupe enfin, où l'on retrouve un échantillon très varié de toutes les couches de la population, met l'accent moins sur le nationalisme que sur l'autodétermination des groupes et sur la notion de participation. L'autodétermination apparaît alors comme un prérequis à une société où tous les groupes partageraient le pouvoir de définir les objectifs de la société. Bien que la plupart des sous-groupes qu'on pourrait classifier dans cette catégorie revendiquent la participation et l'autodétermination, on ne retrouve pas toujours l'idée de nationalisme et en particulier l'idée de séparatisme. Il semble que ce soit plutôt à la marge que l'idéologie de participation se rapproche du nationalisme. Il s'agit là d'un groupe relativement peu important dans le courant du néo-nationalisme bien qu'il s'agisse d'un mouvement relativement important dans l'ensemble de la société.

Bien qu'elle ait baigné dans le contexte nord-américain capitaliste, la société canadienne-française est en voie de passer de la société traditionnelle à la société technocratique sans ellemême connaître une étape importante de pouvoir capitaliste ou de pouvoir ouvrier. Le nationalisme canadien-français dans ses avatars nous permet de bien saisir ce passage et ses caractéristiques. L'analyse du néo-nationalisme nous montre cependant que les jeux ne sont pas complètement faits et qu'à courte échéance l'affrontement entre les technocrates et la petite bourgeoisie économique est inévitable. Leur alliance temporaire dans le parti québecois va finir par se résoudre dans le pouvoir d'un groupe au détriment de l'autre lorsque le succès de ce parti deviendra chose faite.

Divers indices au niveau des autres paliers de la société nous laisseraient croire que le pouvoir finira par appartenir au groupe technocratique. Cependant cette absence de prédominance de la bourgeoisie dans l'histoire de notre peuple va peut-être hâter la réalisation dans notre société d'une véritable structure de participation. La société de participation, en effet, s'oppose 
à la société technocratique comme la société libérale s'oppose à la société traditionnelle. La technocratie s'imposant ici sans avoir à lutter contre le capitalisme sera ainsi moins aguerrie et pour autant plus susceptible de céder devant les pressions de la société de participation.

GÉRALD FORTIN 